\title{
Gastrointestinal bleeding as an initial manifestation in asymptomatic multiple myeloma: A case report and review of the literature
}

\author{
MAOSONG LIN ${ }^{1}$, JIAYI ZHU ${ }^{1}$, HONGZHANG SHEN ${ }^{1}$ and JUNXING HUANG ${ }^{2}$ \\ Departments of ${ }^{1}$ Gastroenterology and ${ }^{2}$ Oncology, Taizhou People's Hospital, Taizhou, Jiangsu 225300, P.R. China
}

Received June 27, 2012; Accepted September 17, 2012

DOI: $10.3892 / \mathrm{ol} .2012 .945$

\begin{abstract}
Multiple myeloma (MM) is an uncommon type of malignant hematological neoplasm which, besides primarily involving the bone marrow, has a potent tendency to involve other organs and to present with various clinical manifestations. Involvement of the gastrointestinal (GI) system, particularly presenting with GI hemorrhage, in the course of MM is extremely rare. In the present study, we report a case of a middle-aged male who initially demonstrated GI bleeding and was finally diagnosed with MM. Further laboratory examinations, including analysis of globulin in the blood, bone marrow aspiration and radiogram, supported this diagnosis. This case may promote the possible involvement of plasma cell myeloma in the differential diagnosis of patients with unexplained and refractory GI bleeding. In this study we also present a thorough review of the literature with regard to the association between MM and GI hemorrhage.
\end{abstract}

\section{Introduction}

Gastrointestinal (GI) bleeding usually originates from peptic ulcers, acute gastric mucosal lesions, esoghageal varices second to hepatic cirrhosis and primary digestive system neoplasms. However, some potent asymptomatic diseases of the blood system manifesting as GI hemorrhage may be clinically ignored. The incidence of gastrointestinal bleeding has been reported as $10-30 \%$ of patients with neoplastic diseases of the hematopoietic organ (1). The pathogenesis of gastrointestinal bleeding in these diseases is usually multifactorial and may be caused by direct malignant hematopoietic cell infiltrations, mucosal changes ensuing from bone marrow suppression,

Correspondence to: Dr Maosong Lin, Department of Gastroenterology, Taizhou People's Hospital, 210 Yingchun Road, Taizhou, Jiangsu 225300, P.R. China

E-mail:1ms0605@163.com

Key words: multiple myeloma, gastrointestinal bleeding, peptic ulcer, hematochezia, hemotological neoplasm immunodeficiency states, infections due to various organisms, or preceding peptic ulcers (1).

Multiple myeloma (MM), also known as plasma cell myeloma, is a malignant hematological neoplasm, characterized by clonal proliferation of plasma cells in the bone marrow and associated with the overproduction of structurally homogeneous immunoglobulins. Anemia, renal failure, skeletal destruction, recurrent infections and hypercalcemia are the most common features of the disease. New diagnostic criteria of MM require the presence of at least $10 \%$ plasma cells on examination of the bone marrow (or biopsy of tissue with monoclonal plasma cells), monoclonal protein in the serum or urine, and evidence of end-organ damage (2). Clinicopathological studies demonstrated that clinically apparent extraosseous manifestations were present in less than $5 \%$ of patients with MM. Liver, spleen and lymph nodes were the most frequent sites of occurrence in approximately two-thirds of these patients, while central nervous system, kidney, skin, pleura, lung, testes, pancreas, thyroid, adrenal glands and omental involvement had also been revealed (3). Involvement of the GI system, particularly demonstrating with GI hemorrhage, in the course of MM is extremely rare.

In this report we describe a case which was finally diagnosed as MM, but was initially manifested as gastrointestinal hemorrhage. We also present a thorough review of the literature with regard to the association between MM and GI bleeding. The study was approved by the Ethics Committee of Taizhou People's Hospital, Jiangsu, China. Written informed consent was obtained from the patient.

\section{Case report}

A 47-year-old male was admitted to the Taizhou People's Hospital (Jiangsu, China) with a two year history of recurrent hematochezia. Two days prior to admission, the patient's symptoms deteriorated with three times the normal blood stool passage. The patient also felt dizzy and experienced palpitations. In April and July 2012, the patient experienced two instances of hematochezia, and at that time, ulcers were identified in the stomach and duodenum through a gastroscopy. Meanwhile, colorectal polyps were identified through a colonoscopy and gastrointestinal barium imaging revealed a normal small intestine. Following acid suppression therapy, the 
patient's stool recovered to normal, and surgery was conducted five years ago to treat the intestinal obstruction.

Upon physical examination, the positive signs included the anemic appearance and a surgical scar on the left side of the abdomen.

At the initial examination, laboratory results were as follows: red blood cells, $1.87 \times 10^{12} / 1\left(4-5.5 \times 10^{12} / 1\right)$; hemoglobin, $102 \mathrm{~g} / 1$ (120-160 g/l); white blood cells, $5.35 \times 10^{9} / 1$ (4-10×109/1); platelets, $242 \times 10^{9} / 1\left(100-300 \times 10^{9} / 1\right)$; and erythrocyte sedimentation rate (ESR), $125 \mathrm{~mm} / \mathrm{h}(10-20 \mathrm{~mm} / \mathrm{h})$. Biochemical examination revealed the following results: total serum protein, $123.1 \mathrm{~g} / 1$ (66-87 g/1); albumin, $25.6 \mathrm{~g} / \mathrm{l}$ (35-54 g/1); globulin, $97.5 \mathrm{~g} / 1$ (20-40 g/l); IgG, $82.4 \mathrm{~g} / 1$ (8-17 g/1); IgA, $0.23 \mathrm{~g} / 1$ (0.7-4.0 g/1); and IgM, $0.34 \mathrm{~g} / 1$ (0.4-2.3 g/1). Lactate dehydrogenase (LDH), electrolytes, glucose, fat and renal function tests were all normal, and the Bence-Jones protein was not detected in the patient's urine.

In the bone marrow aspirate, $20 \%$ plasma cells were identified (Fig. 1). A plain radiogram, shown in Fig. 2, revealed that multiple lytic lesions were present in the skull, while diffuse osteoporosis was present in the ilium, pubis and sciatic and degeneration was present in the thoracic and lumbar vertebra. Furthermore, the capsule endoscopy revealed edema in the gastric mucosa and an ulcer in the duodenum. A gastroscopy and colonoscopy identified no active hemorrhage in the digestive tract. Thus, according to the diagnostic criteria, this patient was finally diagnosed as MM, IgG type, grade III.

Following diagnosis, the patient received systemic chemotherapy with mephalan, prednisone and oral thalidomide, and acquired partial remission. Following 6 months of therapy, bone marrow aspiration revealed that the number of plasma cells was less than 5\%, and globulin and $\mathrm{IgG}$ decreased to $64 \mathrm{~g} / \mathrm{l}$ and $53 \mathrm{~g} / \mathrm{l}$, respectively. To date, no GI bleeding has occurred.

\section{Discussion}

$\mathrm{MM}$ is the second most prevalent hematological malignancy, representing $2 \%$ of all cancer mortalities (4). Although usually restricted to the bone marrow, extramedullary involvement of this disease can occur in up to $20 \%$ of cases. The most common extramedullary site is the upper respiratory tract, including the oropharynx, nasopharynx, nasal cavities, sinuses and larynx $(2,5)$. Cases of MM with GI features are uncommon. Some clinical manifestations have identified nausea, vomiting and colic in $20 \%$ of cases, but hematemesis and melena, in contrast to bleeding from the nose and gums, are rare. The marked infrequency of GI hemorrhage in MM may easily lead the clinician to make an erroneous diagnosis of common peptic ulcer or gastric neoplasm, carcinoma or lymphoma. To the best of our knowledge, only a few cases corresponding to GI bleeding in MM have previously been published.

GI bleeding has been identified in $10-30 \%$ of patients with neoplastic diseases of the hematopoietic organ, including MM. Talamo et al (6) identified 24 patients with involvement of the GI system documented in a database of 2,584 patients with MM of which only two cases presented with GI hemorrhage. Through reviewing the limited literatures associated with GI hemorrhage in MM patients, we speculate that the potent pathogenesis of GI bleeding in MM through several pathways including direct plasma cell infiltrations in the form of

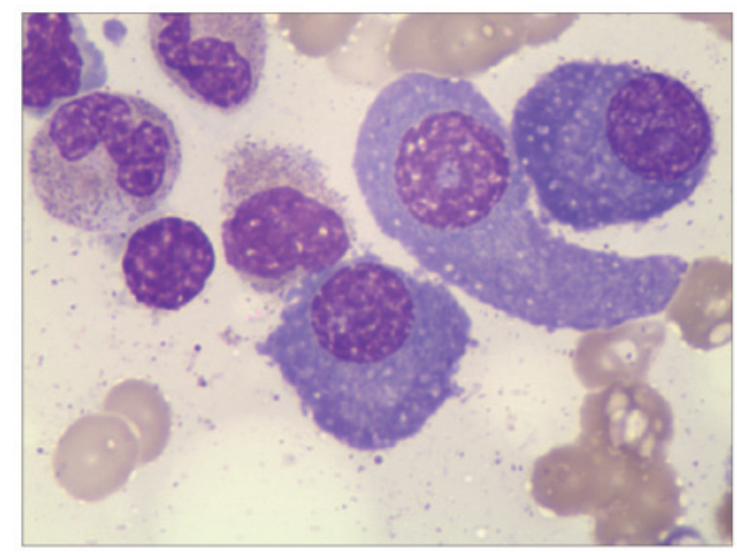

Figure 1. H\&E staining of bone marrow biopsy section showing clusters of abnormal malignant-appearing plasma cells (magnification, x100). H\&E, hematoxylin \& eosin.

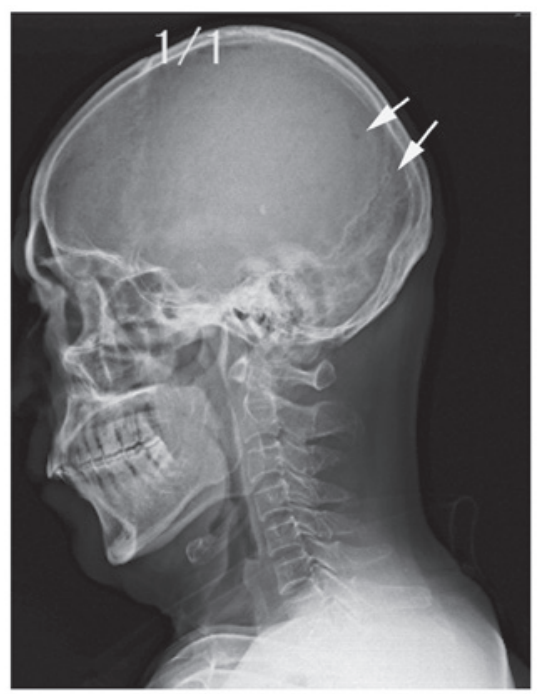

Figure 2. Multiple osteolytic bone lesions in the skull.

plasmacytomas, amyloid infiltration of the gut wall resulting in increased capillary fragility, platelet or coagulation abnormalities, and preceding peptic ulcers may sometimes be secondary to treatment with anti-inflammatory medications and corticosteroids.

Daram et al (7) reported a case of a 53-year-old female diagnosed with MM in 2005. The patient presented with acute upper GI bleeding in the form of hematemesis, and underwent chemotherapy and stem cell transplantation. An emergent esophagogastroduodenoscopy identified a multilobulated, submucosal mass on the proximal corpus along the greater curvature, and a small erosion on the lateral wall of the second portion of duodenum. Further biopsies revealed dense submucosal infiltrates of highly atypical plasmacytic cells in the stomach and duodenum. Additionally, Vicuña Arregui et al (8) and Suvannasankha et al (9) previously demonstrated that plasmacytomas were present in cases with MM. Amyloid was identified in other studies, which revealed another etiology of GI bleeding in MM patients. Chang et al (10) revealed a case of MM presenting with episodes of coffee-ground vomitus or 
massive hematochezia. A tissue biopsy taken at the irregular mucosa beside the protruding vessels in the duodenum, confirmed the diagnosis of GI amyloidosis. Alvares et al (11) also reported the case of a 45-year-old female who presented with hematemesis. Further endoscopy identified nodular lesions and ulcers in the gastric antrum, and histology of the lesions confirmed the diagnosis of amyloidosis. Maza et al (12) reported an uncommon case of rectal bleeding and anemia with polypoid lesions and ulcerations in the colon, as the presenting symptom of amyloidosis and light chain MM. All of these cases mentioned above demonstrated the absence of systemic symptoms of amyloidosis, and non-specific endoscopic findings may make diagnosis difficult. Therefore, we recommend that a diagnosis of amyloidosis-induced GI bleeding should be considered in patients with MM with an obscure hemorrhage. Abnormal hemostasis, specifically the imbalance in function of all major components of the coagulation cascade, may increase the risk of bleeding in patients with MM. DiMinno et al (13) suggest that bleeding occurring in dysproteinemia may be the result of a specific interaction of monoclonal paraproteins with platelets. Saif et al (14) considered that patients with MM may develop bleeding diathesis secondary to a variety of mechanisms. One such mechanism is direct inhibition of fibrin monomer aggregation due to the paraprotein, resulting in prolongation of the thrombin and replitase time.

Finally, the most common etiology may be peptic ulcer, which is usually secondary to treatment with anti-inflammatory medications and corticosteroids. In 2006, Dawson et al (15) reported a case of a 60 -year-old female with MM relapse. The patient presented with hematemesis and melena following salvage chemotherapy with dexamethasone, cyclophosphamide, etoposide and cisplatin, and rescue therapy with single-agent bortezomib. However, in contrast to the cases mentioned above, in the present study, the patient manifested with recurrent GI hemorrhage. The only positive signature was the duodenal ulcer and focus mucosal lesion in a small bowl, without evidence of plasma cell infiltration or amyloidosis, following tissue biopsy. Furthermore, no fatigue, bone pain or recurrent infections were observed, while hyperproteinemia and reversal of the albumin/globulin ratio was identified during the period of present medical history, which fulfilled the criteria for asymptomatic myeloma (16). Additionally, prior to the patient's hematochezia, no non-steroidal anti-inflammatory drugs (NSAIDS) or corticosteroids were administrated and no abnormal hemostasis was identified in laboratory examinations. Thus, primary GI hemorrhage probably attributed to duodenal ulcers and mucosal erosion complicated with MM could explain this case. Following systemic chemotherapy with mephalan and prednisone complying with administration of antacid therapy, the patient acquired partial remission and no recurrence of GI hemorrhage was observed. As a result, since no evidence of related end-organ damage was present, atypical myeloma should be paid more attention during our clinical diagnosis and treatment process.

In conclusion, MM is a systemic disease which may be rarely presented with GI involvement. It should be kept in mind that recurrent GI bleeding is difficult to explain through complete GI work-up using endoscopy, CT-scanning or MRI; thus, it may be related to certain hematopoietic neoplasms, particularly the asymptomatic myeloma involvement, and these patients should be examined further. Furthermore, early diagnosis of this disease has advantages of identifying preceding peptic ulcers and other lesions in the GI tract, as well as providing prophylactic steps to the GI mucosa, which is vulnerable to hemorrhage and further chemotherapy.

\section{References}

1. Yoshida T: Complicated gastrointestinal bleeding of neoplastic diseases of the hematopoietic system. Nihon Rinsho 56: 2405-2410, 1998 (In Japanese).

2. Kyle RA and Rajkumar SV: Multiple myeloma. N Engl J Med 3518: 1860-1873, 2004.

3. Kapadia SB: Multiple myeloma: A clinicopathologic study of 62 consecutively autopsied cases. Medicine 59: 380-392, 1980.

4. Cherpanath TGV, Nieuwdorp M, Hazenberg MD, et al: Large nocturnal eyes causing gastrointestinal bleeding in asymptomatic multiple myeloma. Neth J Med 68: 324-327, 2010.

5. Dolin S and Dewer JP: Extramedullary plasmacytoma. Am J Pathol 32: 83-103, 1956

6. Talamo G, Cavallo F, Zangari M, et al: Clinical and biological features of multiple myeloma involving the gastrointestinal system. Haematologica 91: 964-967, 2006.

7. Daram SR, Paine ER and Swingley AF: Upper gastrointestinal bleeding in a patient with multiple myeloma. Gastroenterology 142: e8-e9, 2012.

8. Vicuña Arregui M, Borobio Aguilar E, Vila Costas JJ, et al: Gastric plasmacytoma as an uncommon cause of upper gastrointestinal bleeding. Gastroenterol Hepatol 31: 217-220, 2008 (In Spanish).

9. Suvannasankha A, Abonour R, Cummings OW and Liangpunsakul S: Gastrointestinal plasmacytoma presenting as gastrointestinal bleeding. Clin Lymphoma Myeloma 8: 309-311, 2008.

10. Chang SS, Lu CL, Tsay SH, et al: Amyloidosis-induced gastrointestinal bleeding in a patient with multiple myeloma. J Clin Gastroenterol 32: 161-163, 2001.

11. Alvares JF, Thomas J, Ramakrishna K, et al: Multiple myeloma with amyloidosis presenting with recurrent gastrointestinal bleeding. Indian J Gastroenterol 22: 196, 2003.

12. Maza I, Vlodavsky E and Eliakim RA: Rectal bleeding as a presenting symptom of AL amyloidosis and multiple myeloma. World J Gastrointest Endosc 2: 44-46, 2010.

13. DiMinno G, Coraggio F, Cerbone AM, et al: Myeloma paraprotein with specificity for platelet glycoprotein ilia in a patient with a fatal bleeding disorder. J Clin Invest 77: 157-164, 1986.

14. Saif MW, Allegra CJ and Greenberg B: Bleeding diathesis in multiple myeloma. J Hematother Stem Cell Res 10: 657-660, 2001.

15. Dawson MA, Polizzotto MN, Gordon A, et al: Extramedullary relapse of multiple myeloma presenting as hematemesis and melena. Nat Clin Pract Oncol 3: 223-226, 2006.

16. Kyle RA and Rajkumar SV: Criteria for diagnosis, staging, risk stratification and response assessment of multiple myeloma. Leukemia 23: 3-9, 2009. 